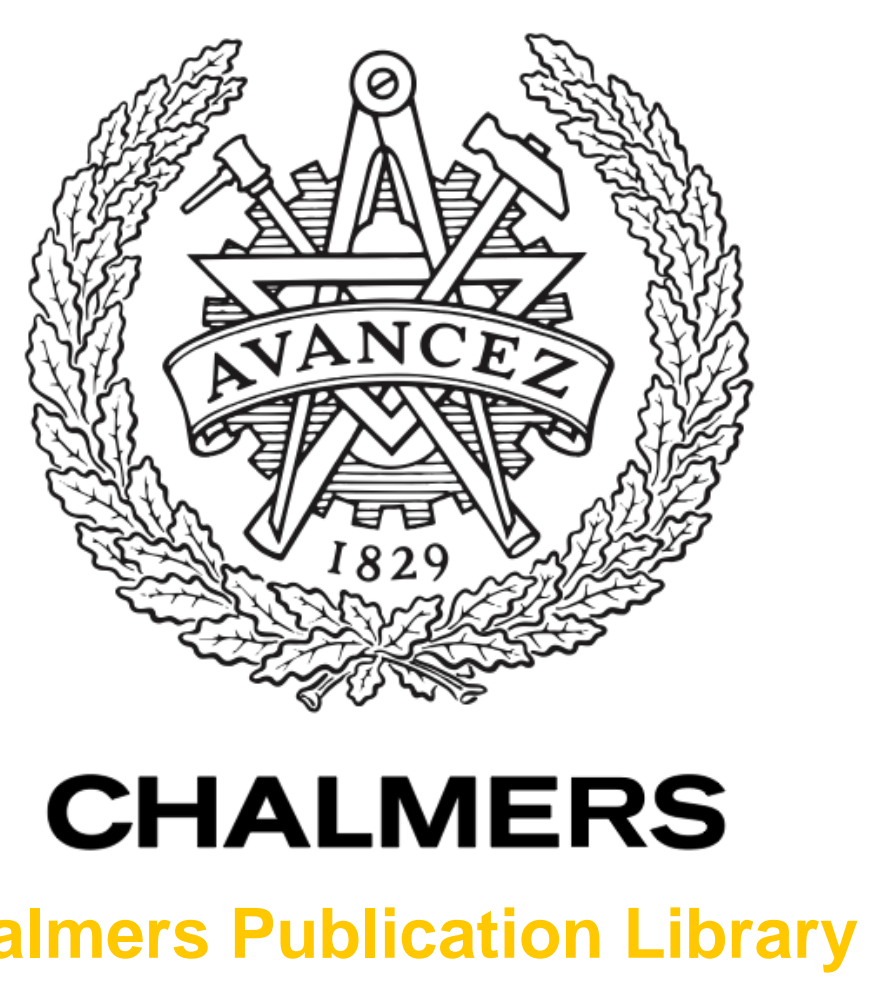

Chalmers Publication Library

\author{
A new compact wideband MIMO antenna - the double-sided tapered self-grounded \\ monopole array
}

This document has been downloaded from Chalmers Publication Library (CPL). It is the author's version of a work that was accepted for publication in:

IEEE Transactions on Antennas and Propagation (ISSN: 0018-926X)

Citation for the published paper:

Al-Rawi, A. ; Hussain, A. ; Yang, J. (2014) "A new compact wideband MIMO antenna - the double-sided tapered self-grounded monopole array". IEEE Transactions on Antennas and

Propagation, vol. 62(6), pp. 3365 - 3369.

http://dx.doi.org/10.1109/TAP.2014.2309985

Downloaded from: http://publications.lib.chalmers.se/publication/199329

Notice: Changes introduced as a result of publishing processes such as copy-editing and formatting may not be reflected in this document. For a definitive version of this work, please refer to the published source. Please note that access to the published version might require a subscription.

Chalmers Publication Library (CPL) offers the possibility of retrieving research publications produced at Chalmers University of Technology. It covers all types of publications: articles, dissertations, licentiate theses, masters theses, conference papers, reports etc. Since 2006 it is the official tool for Chalmers official publication statistics. To ensure that Chalmers research results are disseminated as widely as possible, an Open Access Policy has been adopted.

The CPL service is administrated and maintained by Chalmers Library. 


\section{A New Compact Wideband MIMO Antenna - The Double-sided Tapered Self-grounded Monopole Array}

Ali Al-Rawi, Ahmed Hussain, Jian Yang, Magnus Franzén, Charlie Orlenius, and Ahmed A. Kishk

\begin{abstract}
We present a new compact wideband MIMO (multiple input multiple output) antenna - the double-sided 4-port armtapered self-grounded monopole array, briefly referred to as the butterfly antenna, in the paper. The antenna is very compact with low correlation between ports and high diversity gain. The genetic algorithm optimization scheme has been employed in the design. Simulation results have been verified against measurements. The measured reflection coefficients at all ports are below $-7 \mathrm{~dB}$ over $0.5-9 \mathrm{GHz}$ and below $-4.5 \mathrm{~dB}$ over $0.4-0.5 \mathrm{GHz}$ and 9-15 GHz. The measured correlation coefficients are below 0.4 over 0.4 $15 \mathrm{GHz}$ and lower than 0.1 in most of the frequency band. This new MIMO antenna is developed as a transmit antenna in reverberation chambers, and we believe that it will find more applications in other systems, such as micro base station antennas in wireless communication systems.
\end{abstract}

Index Terms-MIMO antenna, UWB antenna, self-grounded antenna, embedded radiation efficiency, correlation, genetic algorithm optimization.

\section{INTRODUCTION}

MIMO (multiple input multiple output) technology has played an important role in wireless communication systems, because it can increase the data throughput and link range significantly without additional bandwidth or increasing transmit power [1], [2].

MIMO antennas are crucial devices in MIMO systems. Therefore, evaluation of a MIMO antenna by measurement is very important. Among many measurement technologies, the reverberation chamber (RC) has become popular during recent years.

An RC is basically a metal cavity, large enough to support many resonant modes in it. It has been used for EMC (electromagnetic compatibility) testing for decades. Recently, it has been also developed as a measurement instrument for characterization of small antennas used in multipath environments that can be emulated inside an RC [3].

In order to have a wideband MIMO performance measurement of a multiport antenna by using the RC technology, the chamber antenna in an RC must be a multiport one with a wideband performance. Together with the multiport antenna under test, a MIMO system can be emulated in the RC.

The existing Bluetest $\mathrm{RC}$ antenna is a 3-monopole cube antenna operating over $0.7-8 \mathrm{GHz}$. Since many wireless systems operate at a frequency down to $0.4 \mathrm{GHz}$, and our research for radio telescope antennas covers the frequency

Ali Al-Rawi, Ahmed Hussian and Jian Yang are with the Dept of Signals and Systems, Chalmers University of Technology, S-41296 Gothenburg, Sweden. e-mail: A.Al.Rawi@tue.nl, ahmed.hussain@chalmers.se, jian.yang@chalmers.se. Magnus Franzén and Charlie Orlenius are with Bluetest AB, Gothenburg, Sweden. e-mail: magnus.franzen@bluetest.se, charlie.orlenius@bluetest.se. (www.bluetest.se.) Ahmed A. Kishk is with Concordia University, Canada. e-mail: kishk@encs.concordia.ca.

Manuscript received 2013 (a)

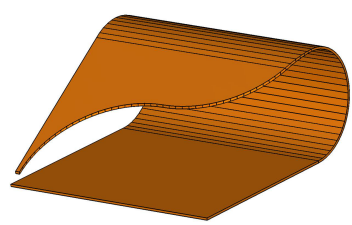

(c)

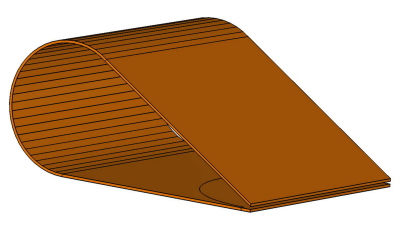

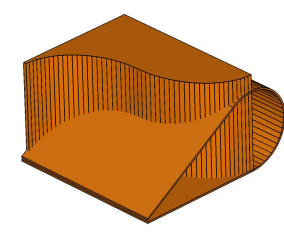

(b)

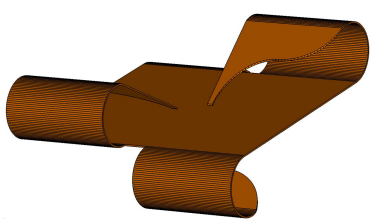

(d)
Fig. 1. Modeling process of the MIMO antenna in CST: (a) self-grounded shape; (b) intersection of tapered geometry with self-grounded shape to make tapered arm; (c) self-grounded tapered monopole; (d) replication and distribution of the monopole to form the MIMO antenna.

band up to $15 \mathrm{GHz}$ [4], [5], an RC operating over $0.4-15$ $\mathrm{GHz}$ is under construction.

The purpose of this work is to develop a MIMO chamber antenna covering $0.4-15 \mathrm{GHz}$ used in RCs, with a compact geometry, high efficiency, a low correlation between ports and a low cost for production.

Many MIMO antennas have been developed for different application scenarios. MIMO dipole arrays in a three dimensional (3D) arrangement were reported in [6], [7]. Planar Eshaped MIMO antennas were proposed in [8], [9]. Similar concept but with ultra-wideband performance was reported in [10]. Planar spiral antennas with circular polarization were proposed as wideband MIMO antennas in [11].

The proposed MIMO antenna in this paper, the double-sided self-grounded monopole array, is based on the self-grounded bow-tie antenna in [12]. The present MIMO antenna has a 3D arrangement in order to have a large radiation coverage with a compact size and at the same time a low correlation between ports over the ultra-wideband of $0.4-15 \mathrm{GHz}$.

A brief report on the current work was presented in [13], while in this paper a detailed description on the design with more simulation and measurement results, especially the RC measurement on radiation performance and diversity gain, is presented.

\section{Modeling And Optimization}

The concept of the proposed antenna is inspired by the self-grounded bow-tie antenna in [12]. In order to have a multiport antenna with a minimum size and a simple feeding structure, a self-grounded monopole is proposed, and four replicas are distributed on both sides of the ground plane to form a MIMO antenna - the double-sided arm-tapered selfgrounded monopole array.

The design concept of the MIMO antenna can be described briefly as follows. When placed vertically above a ground plane, a tapered flat monopole has a similar wideband characteristic to a bicone monopole [14]. In this work, we need four monopoles close to each other with low correlations between them, which is not possible for conventional monopoles. To 


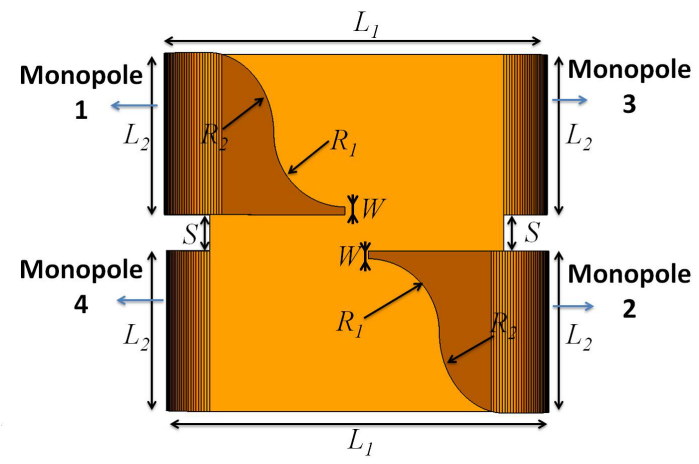

(a)

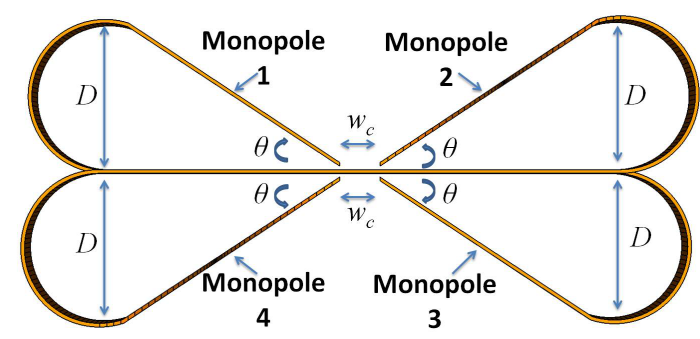

(b)

Fig. 2. Geometry parameters of the antenna: (a) top view; (b) side view.

achieve the required low correlations, the antennas need to be arranged orthogonally to each other. Thus, the following steps are carried out. The tapered flat monopoles are bent with a tilted angle connected to the ground plane, which removes the discontinuities at the arm ends and helps increasing the bandwidth. Two such monopoles are located opposite to each other, and cut to half (at the position of the straight edges that is equivalent of having $\mathrm{H}$-plane cut), which makes the outer edges of the two monopole almost orthogonal in the 3D space (note the tilted arms). This orthogonality makes nearly orthogonal surface currents flowing on each monopole.

In this work, we used the genetic algorithm optimization (GA) scheme [15] together with a commercial EM solver, CST Microwave Studio [16], to achieve a wideband performance. We are working at the moment on the theoretical modeling and analysis of its working mechanism, and the result will be reported in a future publication.

The modeling procedure in CST Microwave Studio can be described as follows. 1) The self-grounded shape is created by intersection of a cylinder to an inclined plane; see Fig. 1(a). 2) The tapered arm of the monopole is modeled by intersection of a cylinder with the self-grounded structure, where the cylinder is made by two circular cylinders of different radii to make different tapering curves; see Fig. 1(b). 3) Then it forms the self-grounded monopole; see Fig. 1(c). 4) The monopole is replicated by mirroring to the ground plane and rotating with axis to have 4 such monopoles; see Fig. 1(d). A discrete port definition is used in CST model during optimization procedure.

Another basic thought behind this array arrangement is to make the radiation patterns of the four antennas different

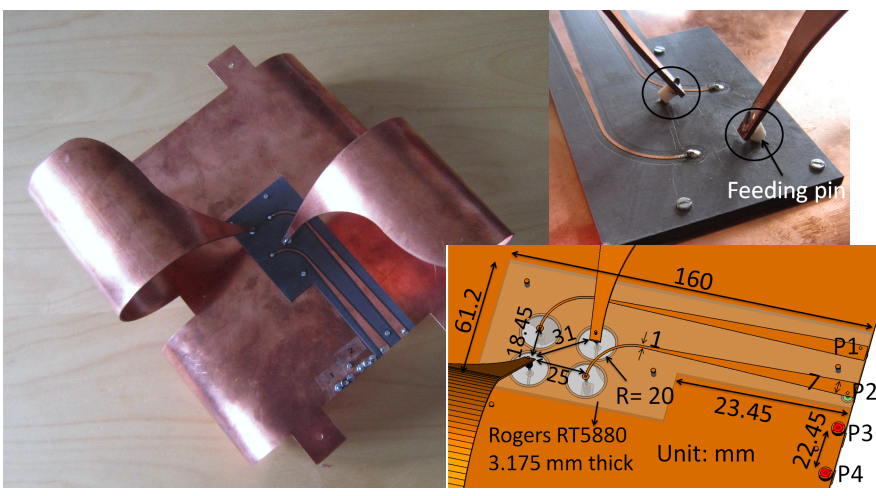

Fig. 3. Manufactured prototype of the proposed MIMO antenna (left), the bending problem of the feeding pins due to the force applied by the antenna arms (top-right), and dimensions of the impedance microstrip line transformer (bottom-right).

(radiating into different directions) with different polarizations, so that the radiation pattern diversity and polarization diversity of the MIMO antenna can be maximized. The simulated and measured diversity gains presented in Sect. IV have verified our thought.

The geometry of the antenna is defined by the parameters of $\left(L_{1}, L_{2}, R_{1}, R_{2}, W, S, D, w_{c}, \theta\right)$, as shown in Fig. 2 .

The optimization goal is to minimize both the reflection coefficients of ports and the mutual couplings between them over $0.4-15 \mathrm{GHz}$, where the cost function values are the highest reflection coefficient of all ports and the highest mutual coupling between them over the band. Therefore, we have a two-objective optimization. The GA Matlab code is based on our previous work in [17], [18]. The initial population of 300 chromosomes is created randomly. Then, a dominating sorting of 15 best chromosomes in each generation produces an elite group of 45 chromosomes for the next generation. Optimization was converged after 5 generations.

When we evaluate the reflection coefficient $S_{11}$ in CST, a port impedance for the discrete port must be decided. A subroutine is implemented in our GA Matlab program so that a search for an optimal port impedance is performed for each chromosome (an individual case of the antenna) to obtain the minimum $S_{11}$ over the whole frequency range. It was found that the optimal port impedance (or the nominal port impedance) of the antenna is between 130-135 Ohms. Therefore, the port impedance of 135 Ohms is decided.

The final optimized antenna, with its dimension parameters listed in Table I, has a total size of $250 \times 250 \times 175 \mathrm{~mm}^{3}$, only $40 \%$ of the volume of the previous monopole cube antenna. This size corresponds to $0.33 \times 0.33 \times 0.23 \lambda_{\text {max }}^{3}$, a very compact MIMO antenna, where $\lambda_{\max }$ is the wavelength at the lowest operating frequency $(0.4 \mathrm{GHz})$ of this antenna.

Since the optimal port impedance of the antenna is 135 Ohms, an impedance transformer from $135 \mathrm{Ohms}$ to 50 Ohms is needed. The microstrip line impedance transformer on Rogers RT5880 board was chosen due to its wideband performance and low Ohmic loss, and the design was done by parametric study. The geometry and the dimensions of the final design of the wideband microstrip line transformer are shown in Fig. 3: a feeding pin inside a Teflon cylinder is connected 


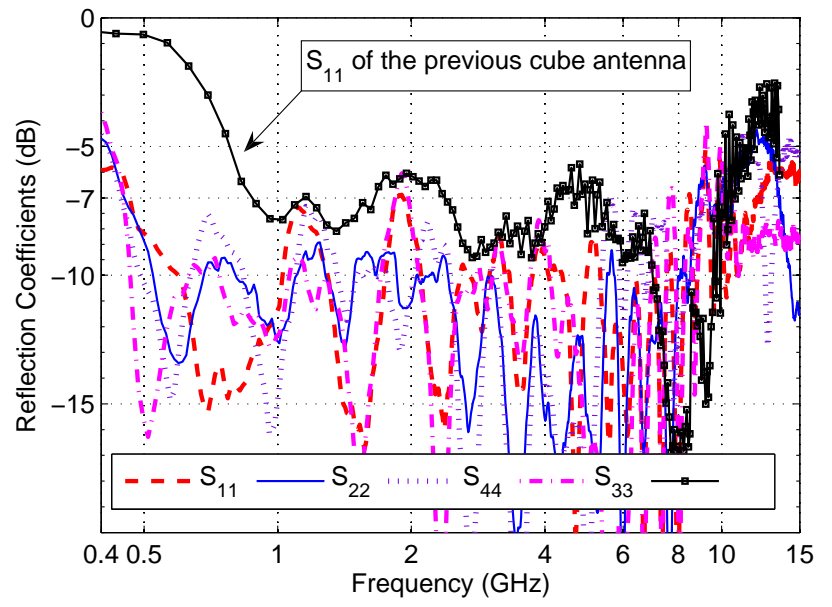

Fig. 4. Measured reflection coefficients of the MIMO antenna.

to the antenna arm above the ground plane at one end and to the microstrip line under the ground plane through a hole at the other end, and then the width of the microstrip line for $135 \mathrm{Ohm}$ characteristic impedance is changed linearly to that for $50 \mathrm{Ohm}$ characteristic impedance.

TABLE I

DIMENSIONS OF THE OPTIMIZED MIMO ANTENNA

\begin{tabular}{c|c||c|c}
\hline \hline Parameter & Value $(\mathrm{mm})$ & Parameter & Value $(\mathrm{mm})$ \\
\hline$L_{1}$ & 250 & $L_{2}$ & 116 \\
\hline$R_{1}$ & 79.5 & $R_{2}$ & 30 \\
\hline$S$ & 20.23 & $W$ & 4.7 \\
\hline$D$ & 87.5 & $W_{c}$ & 15.5 \\
\hline$\theta$ & $55^{\circ}$ & \multicolumn{1}{|l}{} \\
\hline \hline
\end{tabular}

\section{Simulated And Measured Results}

Now, we present the simulated and measured results for final design. The manufactured prototype of the proposed MIMO antenna is shown in Fig. 3.

\section{A. Reflection Coefficient}

The measured reflection coefficient of the antenna is shown in Fig. 4 (the simulated one can be found in [13]). It is found that the measured data has some degradation (below $-4.5 \mathrm{~dB}$ ) above $9 \mathrm{GHz}$ compared to the simulated one. The reason for this is that the feeding pins were bent undesirably by the force from the monopole arms; see the top-right part in Fig. 3. We are looking for a better mechanical solution to it. Nevertheless, from the figure, we can see that the present antenna has much better performance than the previous monopole cube antenna, especially at low frequencies.

\section{B. Mutual Coupling}

Fig. 5 shows the measured mutual couplings between ports, which are below $-15 \mathrm{~dB}$ in the frequency band of $0.4-15 \mathrm{GHz}$ and better than $-20 \mathrm{~dB}$ in most of the band.

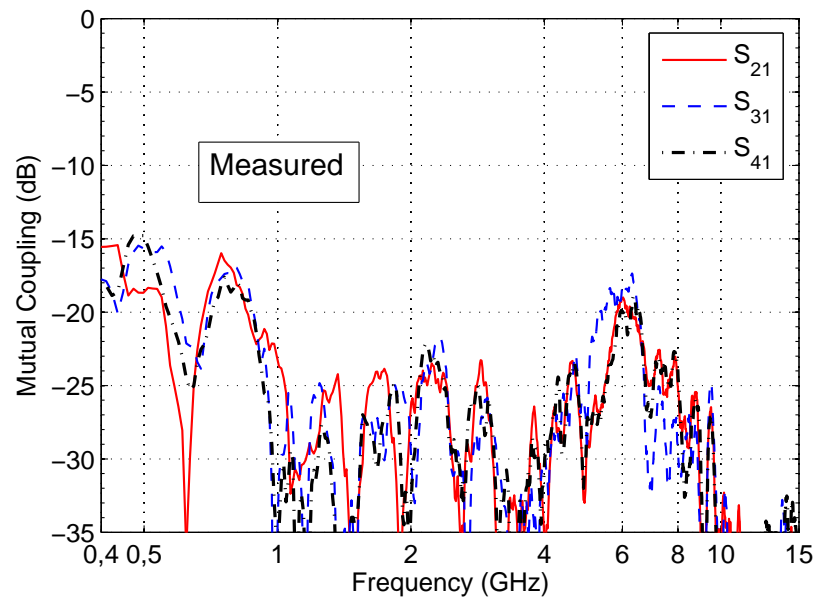

Fig. 5. Measured mutual Couplings of the MIMO antenna.

\section{Polarization Balance}

As mentioned in the previous section, one reason that the configuration of the four self-grounded monopoles is arranged in this way is to have a polarization balance (having two orthogonal components of the E-field) in all radiation directions for a better polarization diversity. We define here the polarization balance $B_{\text {pol }}(\theta, \varphi)$ as

$$
B_{\mathrm{pol}}(\theta, \varphi)=\frac{\sum_{i=1}^{4}\left|E_{\theta}(\theta, \varphi, i)\right|^{2}}{\sum_{i=1}^{4}\left|E_{\varphi}(\theta, \varphi, i)\right|^{2}}
$$

where $E_{\theta}(\theta, \varphi, i)$ and $E_{\varphi}(\theta, \varphi, i)$ are the $\theta$ and $\varphi$ components of the E-field in direction of $(\theta, \varphi)$ when port $i$ is excited and all other ports are terminated, respectively. The value of a perfect polarization balance is $0 \mathrm{~dB}$. In order to present this performance better in the paper, we define the maximum and the minimum of $B_{\text {pol }}$ over $\theta \in\left(0^{\circ}, 180^{\circ}\right)$ in a $\varphi$ plane as

$$
\begin{aligned}
& B_{\text {pol_max }}(\varphi)=\underset{\theta \in\left(0^{\circ}, 180^{\circ}\right)}{\arg \max } B_{\text {pol }}(\theta, \varphi), \\
& B_{\text {pol_min }}(\varphi)=\underset{\theta \in\left(0^{\circ}, 180^{\circ}\right)}{\arg \min } B_{\text {pol }}(\theta, \varphi) .
\end{aligned}
$$

Fig. 6 shows the values of polarization balance in three planes $\left(\varphi=0^{\circ}, 45^{\circ}, 90^{\circ}\right)$ of the present MIMO antenna, based on simulated far-field functions over the frequency band (0.4-15 $\mathrm{GHz}$ ). It can be seen that over the most of frequencies, the polarization balance is between -5 and $5 \mathrm{~dB}$, a good balance over the two orthogonal components.

\section{Reverberation Chamber Measurement}

It is quite time consuming and laborious to measure the far-field function of a MIMO antenna over the whole space in an anechoic chamber. The reverberation chamber, by contrast, provides a fast assessment of the radiation performance for MIMO antennas with a reasonable accuracy. Therefore, we conducted the RC measurement, instead of anechoic chamber, in this work. The used $\mathrm{RC}$ has a size of $1.9 \times 2 \times 1.4 \mathrm{~m}^{3}$ and provides a measurement accuracy of $\pm 0.5 \mathrm{~dB}$ standard deviation over $0.7-8 \mathrm{GHz}$ [3]. For the frequency range of 0.4 $0.7 \mathrm{GHz}$, no verified accuracy data is available for this $\mathrm{RC}$ 


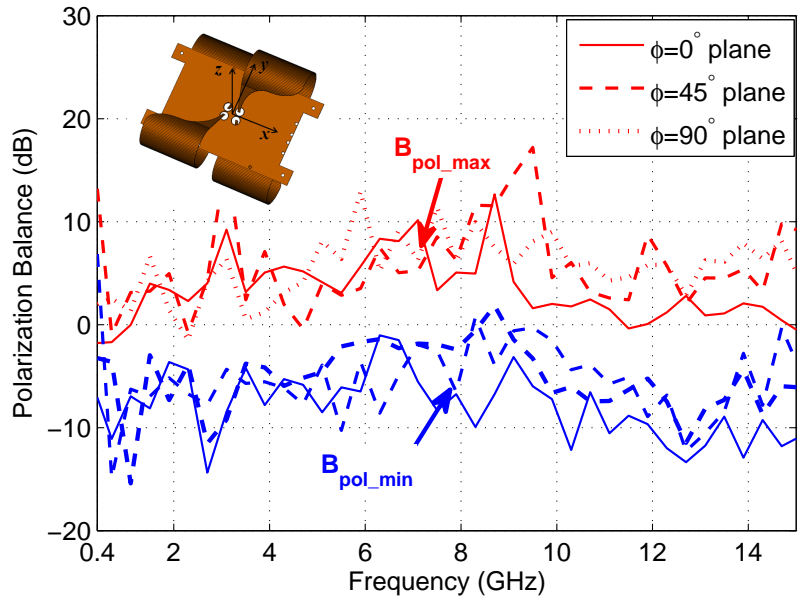

Fig. 6. Calculated polarization balance of the present MIMO antenna.

at the moment. Nevertheless, we still did the measurement evaluation over $0.4-0.7 \mathrm{GHz}$ with this RC. A larger RC is now being under construction to cover the frequencies down to $0.4 \mathrm{GHz}$.

\section{E. Radiation Efficiency}

The total embedded radiation efficiencies measure both the Ohmic loss in the conductive and dielectric parts of the antenna structure and the power loss coupled or absorbed by all ports (Note including input port), which can be expressed for port $i(i=1 \cdots, N)$ by

$$
e_{\text {totembrad }}(i)=\frac{P_{\text {rad }}(i)}{P_{\text {in }}(i)},
$$

where $P_{\text {rad }}(i)$ and $P_{\text {in }}(i)$ are the radiated power from the antenna and the input power to port $i$, respectively, when only port $i$ is excited and all other ports are terminated with loads. We have also

$$
\begin{aligned}
& e_{\text {totembrad }}(i)=e_{\text {decoupling }}(i) e_{\text {embrad }}(i), \\
& e_{\text {decoupling }}(i)=1-\sum_{j=1}^{N}\left|S_{j i}\right|^{2}
\end{aligned}
$$

where $e_{\text {decoupling }}(i)$ accounts for the power loss absorbed by all ports, $e_{\text {embrad }}(i)$ accounts for only the Ohmic loss of the antenna structure, $S_{j i}$ is the S-parameter between ports $i$ and $j$. Then, we have

$$
e_{\text {embrad }}(i)=\frac{e_{\text {totembrad }}(i)}{e_{\text {decoupling }}(i)} .
$$

Fig. 7 shows the simulated and measured embedded radiation efficiencies for all ports. The simulated embedded and total embedded radiation efficiencies are better than $-0.35 \mathrm{~dB}$ and $-1 \mathrm{~dB}$ over $0.5-15 \mathrm{GHz}$, respectively. The measured embedded radiation efficiency is higher than $-0.9 \mathrm{~dB}$ over the whole test band of $0.5-8 \mathrm{GHz}$, and higher than $-0.5 \mathrm{~dB}$ in most of the band. The measured total embedded radiation efficiency is higher than $-1.6 \mathrm{~dB}$ over the whole test band and better than $-1 \mathrm{~dB}$ in most of the band. There is a discrepancy between

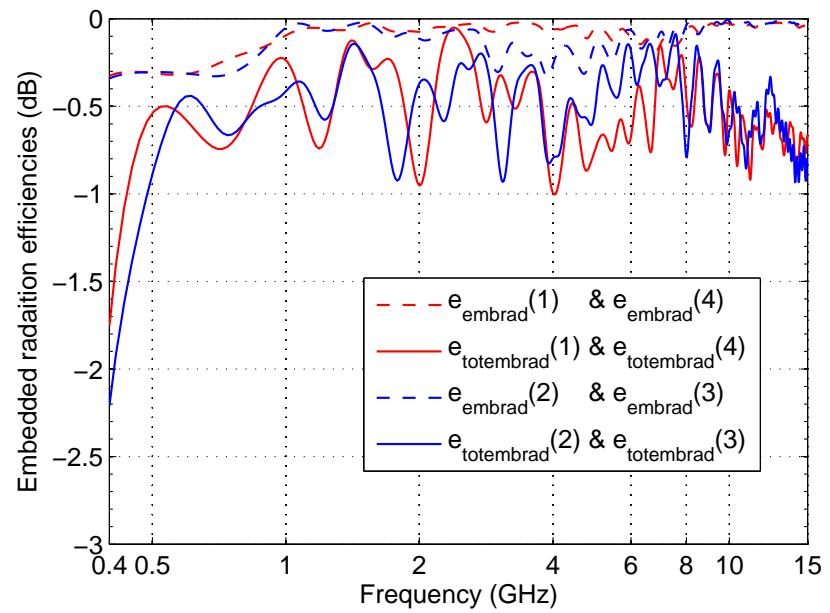

(a)

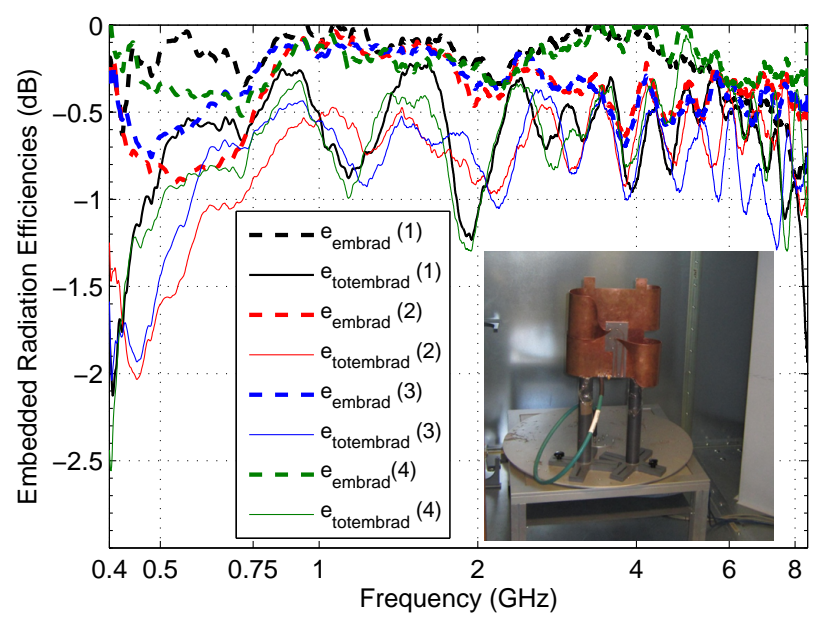

(b)

Fig. 7. Embedded radiation efficiencies of the MIMO antenna: (a) calculated based on measured S parameters; (b) measured by using RC.

measurement and simulation, especially at low frequencies. The reason is, we believe, that the chamber used in this work does not have a size large enough for the frequency range below $0.7 \mathrm{GHz}$. This means that there are not enough numbers of the independent modes inside the chamber at the low frequencies, which leads to a less accurate measurement.

\section{F. Correlation Coefficient}

Fig. 8 is for the measured magnitude of complex correlation coefficients against the calculated one. The calculated correlations are obtained by using formula (4) in [19] and (11) in [20] based on the simulated S-parameters and the simulated radiation efficiencies, where the Ohmic loss of the antenna has been taken into account. The calculated values are below 0.33 and 0.14 over $0.4-0.5 \mathrm{GHz}$ and $0.5-15 \mathrm{GHz}$, respectively. The measured correlation is conducted in the RC directly based on its definition. The measured values are below 0.4 over $0.4-0.7 \mathrm{GHz}$ and below 0.1 over $0.7-8 \mathrm{GHz}$. The difference between measurement and computation is observed to be about 0.1 at the low frequencies, which is considered 


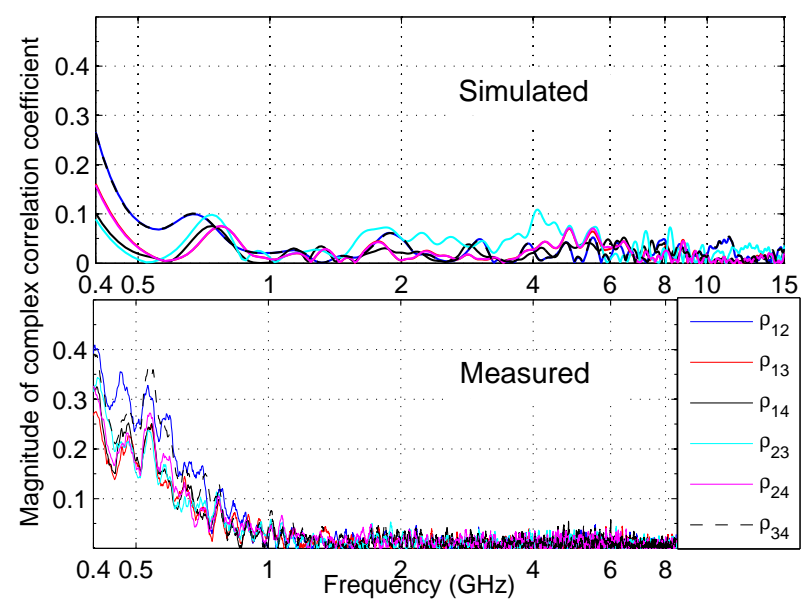

Fig. 8. Magnitude of complex correlation coefficients of the MIMO antenna: (top) simulated by ViRM-lab; (bottom) measured by RC.

to be a good agreement. From the figure, it can be also seen that the repeatability of the measurement is good, since the measured correlation coefficients between all ports are always higher than the simulated one with an almost certain value of 0.1 at $0.4 \mathrm{GHz}$. On the whole, the signals received by this multiport antenna have low correlation, indicating a good diversity performance.

\section{G. Apparent Diversity Gain}

Fig. 9 shows the measured and simulated apparent diversity gain with maximal ratio combining (MRC) scheme for the proposed antenna, used as a 2-, 3-, or 4-port MIMO antenna. The simulation was done by using a ray-based simulation tool called ViRM-lab [21]. A set of 20 incoming waves with a Rayleigh distributed amplitude and uniformly distributed phase, polarization and angle of arrival (AoA) over the whole space was used in the simulation. The CST simulated embedded far-field functions for all ports were exposed to one set of these incoming waves in ViRM-lab, which results in one voltage sample at each antenna port. The cumulative distribution function (CDF) [22] was obtained by collecting 100,000 voltage samples at each antenna port by applying 100,000 such sets of the randomly incoming waves in the simulation. The cumulative distribution function (CDF) can be also obtained directly by the measurement of the antenna in the RC [23]. Based on the simulated and measured CDFs, as shown in Fig. 10, the simulated and measured diversity gain of the antenna with MRC scheme were evaluated according to the definition. The simulated and measured diversity gains agree well overall with each other. The apparent diversity gain is about $11 \mathrm{~dB}, 15 \mathrm{~dB}$ and $17 \mathrm{~dB}$ for the 2-, 3- and 4-port MIMO antenna, respectively, stating a good MIMO performance.

\section{CONCLuSion}

The proposed MIMO antenna has a very compact size, high embedded radiation efficiency, low correlation between the ports and stable diversity gain over a wide band. In addition to

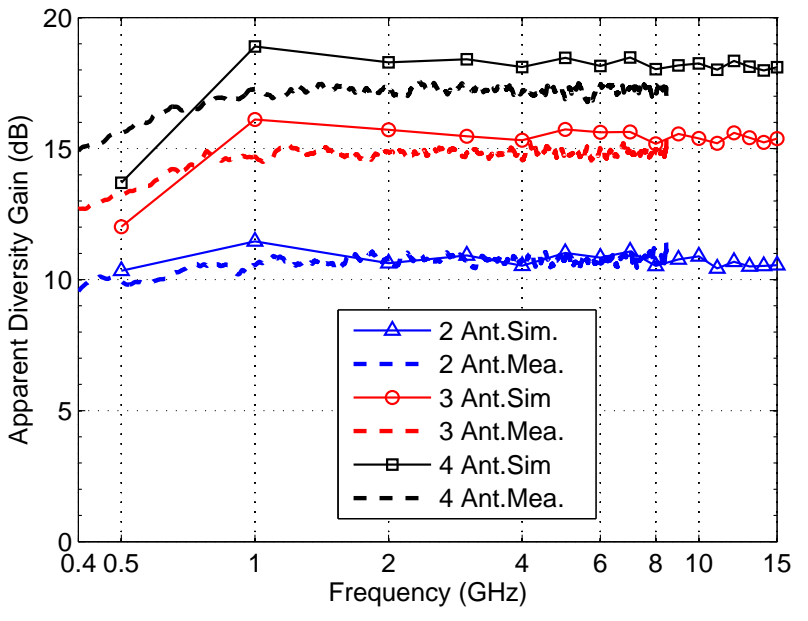

Fig. 9. Measured and simulated apparent diversity gain with maximum ratio combining technique (MRC).

the use in reverberation chambers, we believe that this antenna will find more applications in other MIMO systems.

This antenna is protected by a pending patent [24].

\section{ACKNOWLEDGMENT}

The work was done within the Chase Antenna Systems Excellence Center at Chalmers, which is supported by VINNOVA (Swedish Governmental Agency for Innovation Systems).

\section{REFERENCES}

[1] G. Raleigh and J. Cioffi, "Spatio-temporal coding for wireless communication," IEEE Trans. Commun., vol. 46, no. 3, pp. 357-366, 1998.

[2] V. Tarokh, N. Seshadri, and A. Calderbank, "Space-time codes for high data rate wireless communication: Performance criterion and code construction," IEEE Trans. Inf. Theory, vol. 44, no. 2, pp. 744-765, 1998.

[3] K. Rosengren and P.-S. Kildal, "Radiation efficiency, correlation, diversity gain and capacity of six monopole antenna array for a MIMO system: Theory, simulation and measurement in reverberation chamber," IEE Proc. Microw. Antennas Propag., vol. 152, no. 1, pp. 7-16, 2005.

[4] H. Raza, J. Yang, and A. Hussain, "Measurement of radiation efficiency of multiport antennas with feeding network corrections," IEEE Antennas Wireless Propag. Lett., vol. 11, pp. 89-92, 2012.

[5] J. Yin, J. Yang, M. Pantaleev, and L. Helldner, "The circular Eleven antenna: a new decade-bandwidth feed for reflector antennas with a high aperture efficiency," IEEE Trans. Antennas Propag., vol. 61, no. 8, pp. 3976-3984, 2013.

[6] K. Sulonen, P. Suvikunnas, L. Vuokko, J. Kivinen, and P. Vainikainen, "Comparison of MIMO antenna configurations in picocell and microcell environments," IEEE J. Sel. Areas Commun., vol. 21, no. 5, pp. 703-712, 2003.

[7] B. Getu and J. Andersen, "The MIMO cube - compact MIMO antenna," IEEE Trans. Wireless Commun., vol. 4, no. 3, pp. 1136-1141, 2005.

[8] A. Mallahzadeh, S. Eshaghi, and A. Alipour, "Design of an E-shaped MIMO antenna using IWO algorithm for wireless application at 5.8 GHz," Progress In Electromagnetics Research, vol. 90, pp. 187-203, 2009.

[9] S. Chae, S. Oh, and S. Park, "Analysis of mutual coupling, correlations, and TARC in WiBro MIMO array antenna," IEEE Antennas Wireless Propag. Lett., vol. 6, pp. 122-125, 2007.

[10] S. Lin and H. Huang, "Ultra-wideband MIMO antenna with enhanced isolation,” Microw. Opt. Technol. Lett., vol. 51, no. 2, pp. 570-573, 2009.

[11] C. Waldschmidt and W. Wiesbeck, "Compact wide-band multimode antennas for MIMO and diversity," IEEE Trans. Antennas Propag., vol. 52, no. 8, pp. 1963-1969, 2004. 


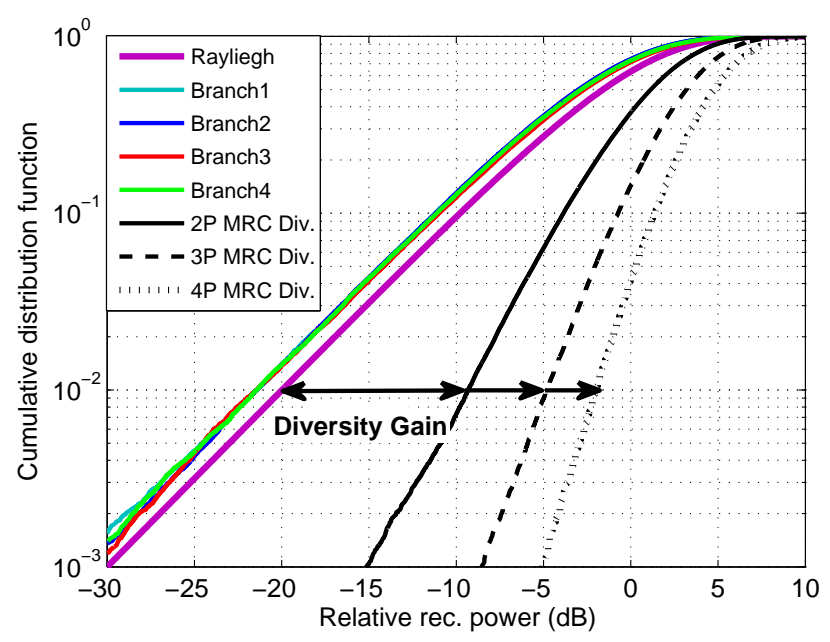

(a)

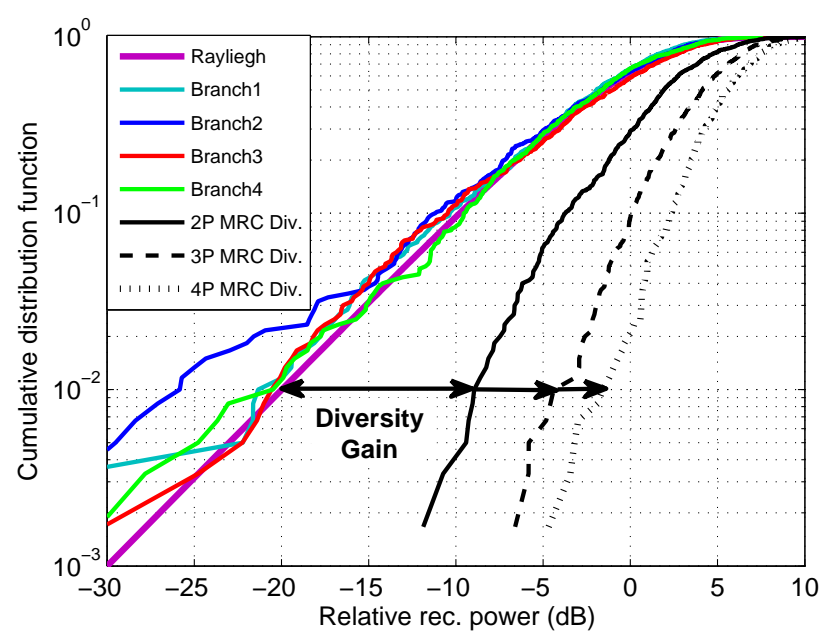

(b)

Fig. 10. The cumulative distribution function (CDF) by using the present MIMO antenna with MRC scheme at $1 \mathrm{GHz}$ (a) simulated by using ViRM-lab at; (b) measured by using RC.

[12] J. Yang and A. Kishk, "A novel low-profile compact directional ultrawideband antenna: the self-grounded Bow-Tie antenna," IEEE Trans. Antennas Propagat., vol. 60, no. 3, pp. 1214-1220, 2012.

[13] A. Al-Rawi, J. Yang, C. Orlenius, and M. Franzen, "The double-sided 4-port Bow-tie antenna: A new compact wideband MIMO antenna," in Proc. 7th Eur. Conf. Antennas Propag. (EUCAP2013), 2013.

[14] A. K. Amert and K. W. Whites, "Miniaturization of the biconical antenna for ultrawideband applications," IEEE Trans. Antennas Propag., vol. 57, no. 12, pp. 3728-3735, 2009.

[15] R. L. Haupt, "An introduction to genetic algorithms for electromagnetics," IEEE Trans. Antennas Propagat., vol. 37, no. 2, pp. 7-15, 1995.

[16] CST Microwave Studio; http://www.cst.com.

[17] J. Yang and P.-S. Kildal, "Optimization of reflection coefficient of large log-periodic array by computing only a small part of it," IEEE Trans. Antennas Propag., vol. 59, no. 6, pp. 1790-1797, 2011.

[18] M. Denstedt, T. Ostling, J. Yang, and P.-S. Kildal, "Tripling bandwidth of hat feed by genetic algorithm optimization," in Proc. IEEE AP-S Symp., 2007, pp. 2197-2200.

[19] I. Salonen and P. Vainikainen, "Estimation of signal correlation in antenna arrays," in Proc. 12th Int. Symp. Antennas (JINA'02), vol. 2, 2002, pp. 383-386.

[20] P. Hallbjorner, "The significance of radition efficiencies when using S-Parameters to calculate the received signal correlation from two antennas," IEEE Antennas Wireless Propag. Lett., vol. 4, pp. 97-99, 2005.
[21] U. Carlberg, J. Carlsson, A. Hussain, and P.-S. Kildal, "Ray based multipath simulation tool for studying convergence and estimating ergodic capacity and diversity gain for antennas with given far-field functions," in Proc. ICECom Conf., 2010, pp. 1-4.

[22] P.-S. Kildal, Foundations of Antennas, A Unified Approach. Studentlitteratur, 2000. ch. 3, pp. 107-108.

[23] X. Chen, P. Kildal, and J. Carlsson, "Comparisons of different methods to determine correlation applied to multi-port UWB Eleven antenna," in Proc. 5th Eur. Conf. Antennas Propag. (EUCAP2011), 2011, pp. 17761780.

[24] J. Yang, A. Al-Rawi, M. Franzén, C. Orlenius, and A. A. Kishk, "Self grounded Antenna Arrangement," Swedish Patent Application SE1251166-3, owened by Gapwaves AB Sweden, 2 October 2012. 